\title{
Research Article \\ Self-Assembling Multifunctional Peptide Dimers for Gene Delivery Systems
}

\author{
Kitae Ryu, ${ }^{1}$ Gyeong Jin Lee, ${ }^{1}$ Ji-yeong Choi, ${ }^{1}$ Taewan Kim, ${ }^{1}$ and Tae-il Kim ${ }^{1,2}$ \\ ${ }^{1}$ Department of Biosystems \& Biomaterials Science and Engineering, College of Agriculture and Life Sciences, Seoul National University, \\ 1 Gwanak-ro, Gwanak-gu, Seoul 151-921, Republic of Korea \\ ${ }^{2}$ Research Institute of Agriculture and Life Sciences, Seoul National University, 1 Gwanak-ro, Gwanak-gu, \\ Seoul 151-921, Republic of Korea
}

Correspondence should be addressed to Tae-il Kim; seal1004@snu.ac.kr

Received 10 May 2015; Accepted 6 September 2015

Academic Editor: Michele Iafisco

Copyright (C) 2015 Kitae Ryu et al. This is an open access article distributed under the Creative Commons Attribution License, which permits unrestricted use, distribution, and reproduction in any medium, provided the original work is properly cited.

Self-assembling multifunctional peptide was designed for gene delivery systems. The multifunctional peptide (MP) consists of cellular penetrating peptide moiety $\left(\mathrm{R}_{8}\right)$, matrix metalloproteinase-2 (MMP-2) specific sequence (GPLGV), pH-responsive moiety $\left(\mathrm{H}_{5}\right)$, and hydrophobic moiety (palmitic acid) $\left(\mathrm{CR}_{8} \mathrm{GPLGVH}_{5}-\mathrm{Pal}\right)$. MP was oxidized to form multifunctional peptide dimer (MPD) by DMSO oxidation of thiols in terminal cysteine residues. MPD could condense pDNA successfully at a weight ratio of 5. MPD itself could self-assemble into submicron micelle particles via hydrophobic interaction, of which critical micelle concentration is about $0.01 \mathrm{mM}$. MPD showed concentration-dependent but low cytotoxicity in comparison with PEI25k. MPD polyplexes showed low transfection efficiency in HEK293 cells expressing low level of MMP-2 but high transfection efficiency in A549 and C2C12 cells expressing high level of MMP-2, meaning the enhanced transfection efficiency probably due to MMP-induced structural change of polyplexes. Bafilomycin A1-treated transfection results suggest that the transfection of MPD is mediated via endosomal escape by endosome buffering ability. These results show the potential of MPD for MMP-2 targeted gene delivery systems due to its multifunctionality.

\section{Introduction}

Lots of peptides have been developed and used for gene delivery systems due to their advantages [1-3]. Building blocks of peptides, amino acids, are nontoxic and almost nonimmunogenic endogenous molecules possessing various functional side chains with the diversity for designing gene delivery carriers. Peptides can be synthesized by wellestablished solid-phase chemistry in sequence-controlled manner, yielding almost monodispersed products. Peptides also can interact with cellular molecules or components, which induce biologically important events such as recognition of RGD peptide by integrin receptors [4].

Particularly, cellular penetrating peptides such as penetratin, Tat sequence, or oligoarginine have been extensively examined in gene delivery field because they can form nanosized complexes with nucleic acids due to their cationic property and possess high cellular uptake efficiency [5-9].
Besides oligopeptide forms, bioreducible cellular penetrating peptides which are linked via disulfide bonds, also have been developed for gene delivery systems [10-13]. They can be degraded in reductive environment such as cytosol, showing controlled release of nucleic acids and low cytotoxicity [14].

In this work, we designed a self-assembling multifunctional peptide (MP, $\mathrm{CR}_{8} \mathrm{GPLGVH}_{5}$-Pal) in order to combine advantages of several functional peptides for gene delivery systems. It is expected that $R_{8}$ moiety would condense pDNA into polyplex particles and facilitate the cellular uptake of the polyplexes [15]. GPLGV is a substrate sequence of matrix metalloproteinase-2 (MMP-2), which is one of the collagenases overexpressed from several tumor cells and plays important roles in tumor progression and metastasis [16]. It was introduced to cause structural change of the polyplexes for efficient cellular uptake, responding to MMP-2 of tumor cells. Many studies reported the potential of MMPcleavable drug/gene delivery carriers for targeting tumor cells 
$[17,18] . \mathrm{H}_{5}$ moiety was employed for endosome buffering ability of imidazole groups to escape from endosomes after cellular uptake $[19,20]$. Palmitic acid at C-terminal would induce micelle formation via self-assembly of the peptide by hydrophobic interaction. $\mathrm{H}_{5}$ moiety also would contribute to the micelle formation because of hydrophobicity at neutral $\mathrm{pH}$. In addition, we cross-linked this peptide to dimer (MPD) for improvement of micelle formation and performed characterizations to examine the potential of MPD for gene delivery systems.

\section{Materials and Methods}

2.1. Materials. Multifunctional peptide $\left(\mathrm{CR}_{8} \mathrm{GPLGVH}_{5}-\mathrm{Pal}\right)$ was purchased from GL Biochem (Shanghai, China). Thiazolyl Blue Tetrazolium Bromide (MTT reagent), agarose, and 5,5'-dithiobis(2-nitrobenzoic acid) (Ellman's reagent) were purchased from Sigma-Aldrich (St. Louis, MO). Dimethyl sulfoxide (DMSO) was purchased from Merck (Germany). Luciferase assay system was purchased from Promega (Madison, WI). BCA protein assay kit was purchased from Pierce (Rockford, IL). Fetal bovine serum (FBS), 0.25\% trypsin-EDTA, Dulbecco's phosphate buffered saline (DPBS), and Dulbecco's modified Eagle's medium (DMEM) were purchased from Invitrogen (Carlsbad, CA). The luciferase reporter gene-encoding plasmid DNA, pCN-Luci, was amplified in Escherichia coli DH5 $\alpha$ and isolated by Nucleobond Xtra Midi kit (Macherey-Nagel, Germany). MMP-2 enzyme was purchased from Calbiochem (San Diego, CA). All other chemicals were purchased and used without any further purification.

2.2. Synthesis of Peptide Dimers. Multifunctional peptide dimer (MPD) was synthesized by using DMSO autooxidation reaction [21]. Multifunctional peptide (MP) was dissolved in DMSO $(0.5 \mathrm{wt} \%)$ and the solution was stirred under nitrogen gas at room temperature for $24 \mathrm{~h}$. After oxidation reaction, synthesized MPD was purified by diethyl ether precipitation and dried under vacuum at room temperature overnight. Finally, sticky and transparent product was obtained.

2.3. Ellman's Assay. Ellman's assay was carried out to confirm the synthesis of MPD by measuring residual thiols after dimerization of MPM. MPD was dissolved in DMSO (0.2 mM). $50 \mu \mathrm{L}$ of Ellman's reagent solution (DMSO, $4 \mathrm{mg} / \mathrm{mL}$ ) was added to $250 \mu \mathrm{L}$ of sample. After $15 \mathrm{~min}$ of incubation, the absorbance at $499 \mathrm{~nm}$ was measured by a microplate reader (Synergy H1, BioTek, USA). Thiol amount of MPD was calculated based on calibration curve.

2.4. Agarose Gel Electrophoresis. DNA condensation ability of MPD was identified by agarose gel electrophoresis assay. Agarose gel $(0.7 \mathrm{wt} \%)$ containing ethidium bromide was prepared in Tris-Acetate-EDTA (TAE) buffer. The MPD polyplexes $(0.5 \mu \mathrm{g}$ pDNA) having various weight ratios $(0.5,1,2$, 5 , and 10 ) were prepared. After $30 \mathrm{~min}$ of incubation at room temperature, electrophoresis was carried out for $15 \mathrm{~min}$ at
$100 \mathrm{~V}$ (Mupid-2plus, Takara Bio Inc., Japan). The locations of pDNA bands were observed by UV illuminator (ChemiDoc XRS+ gel documentation system, Bio-Rad, Hercules, CA).

2.5. Critical Micelle Concentration (CMC) Measurements. In order to characterize CMC of MPD, pyrene was selected as a model drug as previously reported [22]. $0.2 \mathrm{mM}$ of pyrene solution was prepared by dilution of pyrene/ethanol solution. $3 \mu \mathrm{L}$ of pyrene solutions was added to vials and dried under vacuum overnight in dark condition. After ethanol evaporation, $1 \mathrm{~mL}$ of MPD solution with various concentrations was added to the vials, giving the final pyrene concentration of $6 \times 10^{-7} \mathrm{M}$. The MPD/pyrene solution was sonicated for $10 \mathrm{~min}$ and incubated with gentle mixing at room temperature for $24 \mathrm{~h}$. Absorbance was measured by UV/Vis spectrometer (Optizen POP BIO, Mecasys, Korea) using quartz cuvettes in the range of 200 to $400 \mathrm{~nm}$. The total absorbance was calculated by sum of four absorbance points $(242,272,320$, and $336 \mathrm{~nm})$. CMC of the MPD sample was set to the center of the sigmoid.

2.6. Average Particle Size and Zeta-Potential Measurements. Average sizes and Zeta-potential values of MPD and MPD polyplexes were measured by Zetasizer Nano ZS (Malvern Instruments, UK) with $\mathrm{He}-\mathrm{Ne}$ laser beam $(633 \mathrm{~nm})$ at $25^{\circ} \mathrm{C}$, respectively. $1 \mathrm{~mL}$ of $\mathrm{MPD}(0.01$ and $0.1 \mathrm{mM})$ and $\mathrm{MPD}$ polyplex solutions ( $5 \mu \mathrm{g}$ pDNA, weight ratios: 2,10 , and 20 ) was prepared and used for analysis. Sizes of MPD polyplexes after active MMP-2 cleavage were also examined. MPD polyplexes ( $1 \mu \mathrm{g}$ pDNA, weight ratio: 20$)$ were prepared as described above. The polyplex solution was added to active MMP-2 containing PBS buffer ( $\mathrm{pH} 7.4,10 \mu \mathrm{g} / \mathrm{mL}$ MMP-2) and the mixture was incubated at $37^{\circ} \mathrm{C}$ for 4 hours with gentle shaking. After treatment, the sizes of the polyplexes sample were analyzed by Zetasizer Nano ZS. Average sizes and Zetapotential values of MPD and MPD polyplexes were measured 3 times.

2.7. Atomic Force Microscopy (AFM). The morphologies of MPD particle structures were observed by using atomic force microscopy (Park Systems, Korea). $100 \mu \mathrm{L}$ of MPD aqueous solution samples was prepared $(0.01$ and $0.1 \mathrm{mM})$. $10 \mu \mathrm{L}$ sample aliquots were dropped to fresh mica surface by pipette carefully and then excess of sample was removed by absorption of filter papers from the corner of the mica. The mica samples were dried at room temperature for $24 \mathrm{~h}$ and used for AFM observation. The imaging was set to noncontact tapping mode and the scanning rate was $1 \mathrm{~Hz}$.

2.8. Cell Culture. Human lung adenocarcinoma epithelial cells (A549), human transformed primary embryonal kidney epithelial cells (HEK293), and mouse myoblast cells (C2C12) were grown in DMEM supplemented with 10\% FBS and $1 \%$ penicillin/streptomycin $(\mathrm{P} / \mathrm{S})$ in humidified atmosphere containing $5 \% \mathrm{CO}_{2}$ at $37^{\circ} \mathrm{C}$. 
2.9. Cytotoxicity. Cytotoxicity of MPD was characterized by MTT assay. Cells were seeded into 96-well cell culture plates in $100 \mu \mathrm{L}$ of DMEM (10\% FBS, 1\% P/S) at a density of $1 \times 10^{4}$ cells/well. After $24 \mathrm{~h}$, the cells were treated with $100 \mu \mathrm{L}$ of peptide solutions for $4 \mathrm{~h}$. PEI25k was used as a control. Then, the fresh media were exchanged and further incubated for $24 \mathrm{~h}$. After incubation, $25 \mu \mathrm{L}$ of MTT stock solution $(2 \mathrm{mg} / \mathrm{mL}$ in DPBS) was added to each well and the media were carefully removed after $2 \mathrm{~h}$. The formazan crystals formed by proliferating cells were dissolved with $150 \mu \mathrm{L}$ of DMSO and the absorbance was measured at $570 \mathrm{~nm}$ using a microplate reader (Synergy H1, BioTek, USA). Results were presented as relative cell viabilities (RCV, percentage values relative to value of untreated control cells). All experiments were performed in quadruplicate.

2.10. Transfection Experiments. The transfection efficiency of MPD was examined by measuring luciferase transgene expression. Cells were seeded into 24-well cell culture plates in $500 \mu \mathrm{L}$ of DMEM $(10 \% \mathrm{FBS}, 1 \% \mathrm{P} / \mathrm{S})$ at a density of $5 \times 10^{4}$ cells/well. After $24 \mathrm{~h}$, the media were exchanged to serumfree media and MPD polyplex solutions (weight ratios: 5 , 10, 20, and 30) were added to cells. PEI25k polyplex (weight ratio: 1) was used as a control. After $4 \mathrm{~h}$ of incubation, the transfection medium was replaced with fresh DMEM with $10 \%$ FBS. The cells were further incubated for 2 days and rinsed with $240 \mu \mathrm{L}$ of DPBS twice. The cells were lysed with $120 \mu \mathrm{L}$ of lysis buffer and shaken for $30 \mathrm{~min}$ at room temperature. The cell lysates were centrifuged for $30 \mathrm{~min}$ at $15000 \mathrm{~g}$ and $20 \mu \mathrm{L}$ of supernatants was used for luciferase assay. Luciferase activities were measured using luciferase assay reagent on a microplate reader (Synergy H1, BioTek, USA). The total cellular proteins in cell lysates were quantified by BCA protein assay kit. The final transfection efficiency results were presented in terms of RLU/mg cellular protein. All experiments were performed in triplicate.

2.11. Transfection Experiments with Bafilomycin A1. In order to characterize the endosome buffering ability and transfection mechanism of MPD, transfection experiments with Bafilomycin A1 treatment were carried out. A549 cells were seeded as explained above. $200 \mathrm{nM}$ of Bafilomycin A1 solutions was pretreated for $10 \mathrm{~min}$ before transfection. MPD polyplexes were prepared with weight ratio of 10, 20, and 30. PEI25K was used as a positive control. Polyplex solutions were treated to the cells for $4 \mathrm{~h}$ and the media were exchanged with fresh DMEM with 10\% FBS. After $48 \mathrm{~h}$ of further incubation, transfection efficiency was examined as described above. Final results were presented in terms of relative transfection efficiency (transfection efficiency with Bafilomycin Al/transfection efficiency without Bafilomycin A1).

\section{Results and Discussion}

3.1. Characterization of MPM and MPD. MP consists of cellular penetrating peptide moiety $\left(\mathrm{R}_{8}\right)$, matrix metalloproteinase-2 (MMP-2) specific sequence (GPLGV),

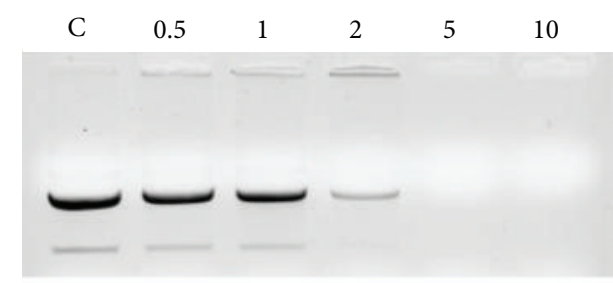

FIgURE 1: Agarose gel electrophoresis result of MPD polyplexes. C: pDNA only. Numbers mean weight ratios of the polyplexes.

pH-responsive moiety $\left(\mathrm{H}_{5}\right)$, and hydrophobic moiety (palmitic acid) $\left(\mathrm{CR}_{8} \mathrm{GPLGVH}_{5}-\mathrm{Pal}\right)$. Cellular penetrating moiety was introduced for condensation of pDNA by electrostatic interaction of cationic arginine residues and for enhancement of cellular uptake. $\mathrm{pH}$-responsive moiety $\left(\mathrm{H}_{5}\right)$ was introduced for facilitation of endosomal escape of polyplexes after cellular uptake by proton sponge effect. Hydrophobic palmitic acid was introduced to C-terminal of MP in order to induce the formation of micelle structure by hydrophobic interaction. $\mathrm{R}_{8}$ and other functional blocks were linked with MMP-2 specific sequence, which may lead to structural change of polyplexes by cleavage of the sequence with exposure to MMP-2.

The structure of MP was confirmed by the manufacturer. MP was dimerized to synthesize MPD by DMSO oxidation via disulfide bond formation between cysteine thiols at $\mathrm{N}$ terminal. MP was insoluble in water but the synthesized MPD after oxidation was soluble in water, probably due to the facilitated formation of micelle structure by strengthened hydrophobic interaction of MPD. It is thought that disulfide bond formation between MP could cause the proximity of hydrophobic moieties (histidine moieties and palmitic acid) and ease of micelle structure formation by structural restriction.

The synthesis of MPD was examined by quantification of remaining thiols of MPD product. After DMSO oxidation, it was found that only 0.3 mole $\%$ of thiol was detected in MPD product, which means that almost MP could be dimerized to synthesize MPD.

3.2. Agarose Gel Electrophoresis. pDNA condensing ability of MPD was investigated by agarose gel electrophoresis. As shown in Figure 1, weak migration of pDNA was still observed at a weight ratio of 2 . However, MPD could retard pDNA completely at a weight ratio of 5 , which means that pDNA was well condensed by MPD. Therefore, it was confirmed that MPD could condense pDNA, due to its cationic $\mathrm{R}_{8}$ moiety.

3.3. CMC Measurement. CMC of MPD was measured in order to confirm the formation of micelle structure by MPD. MPD solutions with various concentrations were incubated with pyrene and absorbances from specific peaks from pyrene were summed. It was reported that absorbance values sum from 4 specific peaks of pyrene would be increased under hydrophobic environment such as core part of micelle 


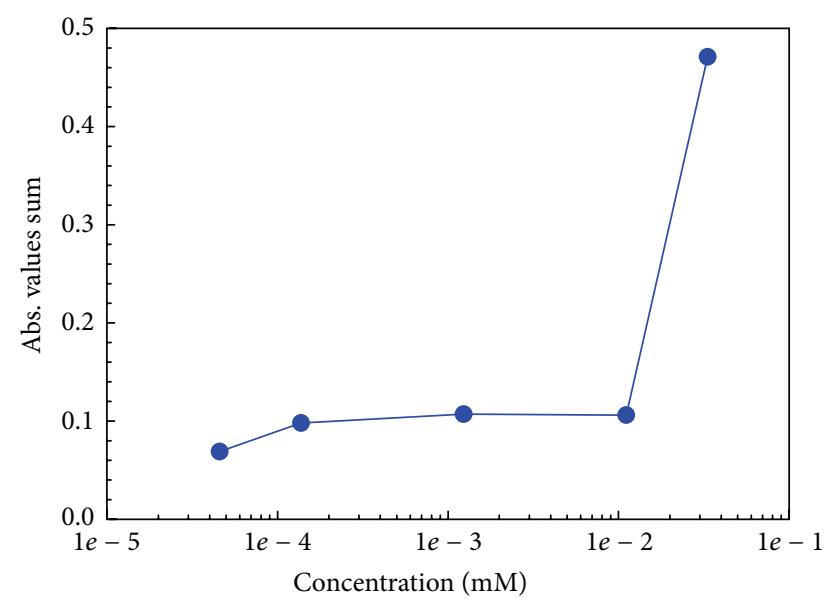

Figure 2: CMC measurement result of MPD micelle.

structures [22]. In Figure 2, absorbance values sum of pyrene was abruptly increased from $0.01 \mathrm{mM}$ of MPD. This result shows that MPD could form micelle structure as expected in aqueous medium and CMC of MPD is about $0.01 \mathrm{mM}$.

\subsection{Characterization of MPD Micelles and MPD Polyplexes.} Average sizes and Zeta-potential values of MPD micelles and MPD polyplexes were measured by Zetasizer (Figure 3). It was found that MPD micelles showed submicron average size at $0.01 \mathrm{mM}$ and the size was decreased to about $470 \mathrm{~nm}$ at $0.1 \mathrm{mM}$, which means the formation of compact and stable micelle structure due to increase of MPD molecules (Figure 3(a)). Zeta-potential values of MPD micelles were also increased from $10.6 \mathrm{mV}$ to $29.2 \mathrm{mV}$, probably due to the additional incorporation of cationic MPD molecules into the micelle structures.

The morphology of MPD micelles was also observed by AFM. They showed heterogeneous structures containing small sphere or large cylinder structures at $0.01 \mathrm{mM}$, which explains large variation of average size measurement results (Figure $4(\mathrm{a})$ ). At $0.1 \mathrm{mM}$, MPD micelles displayed more homogenous morphologies of spherical structures (Figure 4(b)). Greater height of particles was found at $0.1 \mathrm{mM}$ $(30-60 \mathrm{~nm})$ than at $0.01 \mathrm{mM}(5-8 \mathrm{~nm})$, probably due to the increased assembly of MPD molecules into micelle structures at a high concentration.

In the case of MPD polyplexes, average size was $230 \mathrm{~nm}$ at a weight ratio of 2 and increased to about 2-3 $\mu \mathrm{m}$ over weight ratios of 20 (Figure 3(b)). However, Zeta-potential values of MPD polyplexes were found to be negative $(-30.1 \mathrm{mV})$ at a weight ratio of 2 and to be increased to almost zero over weight ratios of 20. Considering Zeta-potential values, it is thought that MPD polyplexes could form large aggregates via hydrophobic interaction at high weight ratios due to the neutralization of surface charges by interaction of cationic MPD micelles with anionic pDNA. It is also elucidated that negatively charged and small MPD polyplex could be partially formed via surface coating of anionic pDNA, which is consistent with agarose gel electrophoresis result.
3.5. Cytotoxicity Measurement. The cytotoxicity of MPD was examined by MTT assay in various cell lines (A549, C2C12, and HEK293). As shown in Figure 5, control agent, PEI25k, exhibited significant cytotoxicity. The viability of PEI25ktreated cells was less than $20 \%$ even at a concentration of $20 \mu \mathrm{g} / \mathrm{mL}$. However, the viability of MPD-treated cells was more than $80 \%$ at the same concentration in all three cell lines. This result means that cytotoxicity of MPD was low at this condition, although it was found to be concentrationdependent.

3.6. Transfection Experiments of MPD Polyplexes. Transfection efficiency of MPD polyplexes was investigated by measurement of luciferase transgene expression in A549, $\mathrm{C} 2 \mathrm{C} 12$, and HEK293 cell lines (Figure 6). It is known that A549 and C2C12 cells express high levels of MMP-2 but HEK293 does not [23-25]. MMP-2 is a family of collagenases which is important for proliferation and migration of cancer cells. Therefore, we performed transfection experiment in various cell lines in order to examine the effect of MMP2 expression on transfection of MPD containing MMP-2 specific sequence. Interestingly, MPD showed high transfection efficiency comparable to PEI25k in A549 and C2C12 cells expressing high level of MMP-2. On the other hand, transfection efficiency of MPD was found to be low (dozens to hundreds lower than that of PEI25k) in HEK293 cells, even though HEK293 cells are known to be well-transfected cells and transfection efficiency of PEI25k in HEK293 cells is about 10-fold higher than that in other cells. This result means that MPD possess the potential for gene delivery systems, especially targeting MMP-2 expressing cancer cells. In addition, the average sizes of the polyplexes were measured in the presence of MMP-2 in order to examine the effect of MPD polyplexes structural change on the transfection efficiency (Figure 6(d)). The size of the polyplexes was found to be largely decreased in the presence of MMP-2. This result shows that the large polyplex aggregates could be converted to smaller polyplex particles by MMP-2 cleavage, probably leading to enhanced cellular uptake and transfection efficiency. Although further study will be required for revealing the detailed mechanism, one explanation is that the structural change of MPD polyplexes by cleavage of MMP-2 such as exposure of cellular penetrating moiety $\left(R_{8}\right)$ may facilitate the cellular uptake of MPD polyplexes for efficient transfection (Scheme 1). On the contrary, in HEK293 cells, MPD polyplexes would not undergo structural change, maintaining large aggregate structures with zero Zeta-potential values, which are not proper for transfection.

3.7. Transfection Experiments with Bafilomycin A1. Transfection experiments were also performed in the presence of Bafilomycin Al in order to examine the endosome buffering ability of MPD and the transfection mechanism. Bafilomycin Al is an inhibitor of vacuolar type ATPase, which can suppress the proton influx into endosome and disturb the proton pump effect of endosome buffering moiety, finally leading to the decrease of transfection efficiency [26, 27]. 


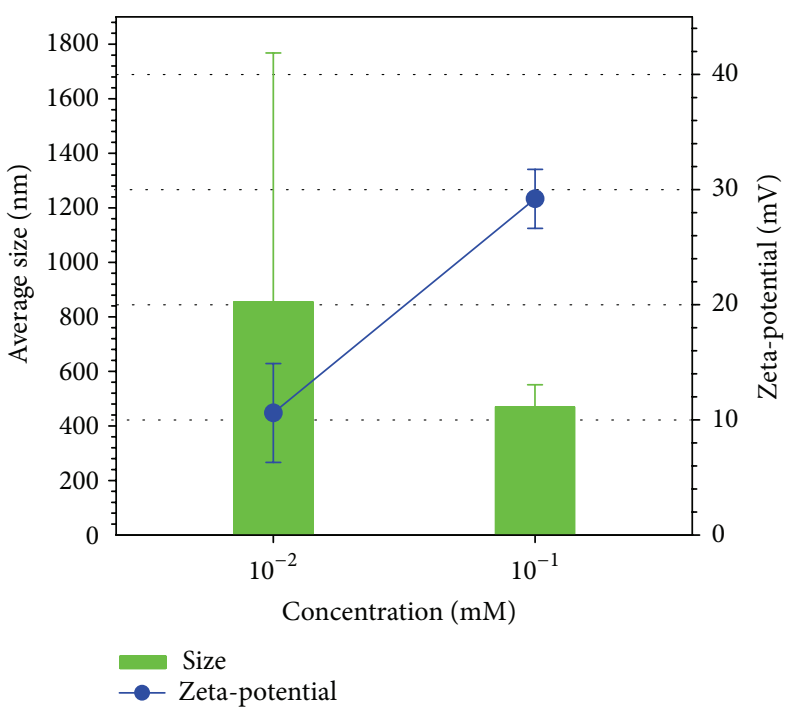

(a)

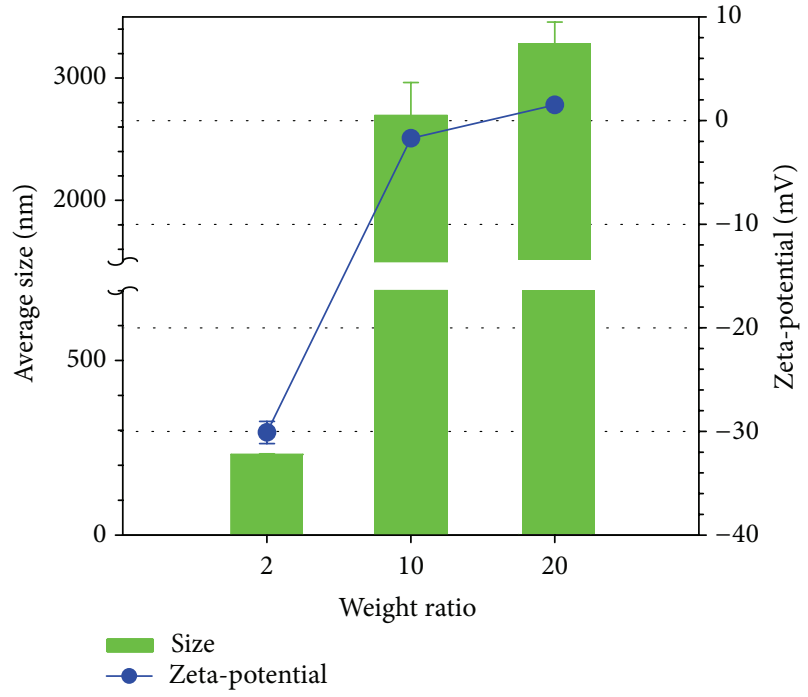

(b)

FIGURE 3: Average sizes and Zeta-potential values measurement results of MPD micelles (a) and MPD polyplexes (b).
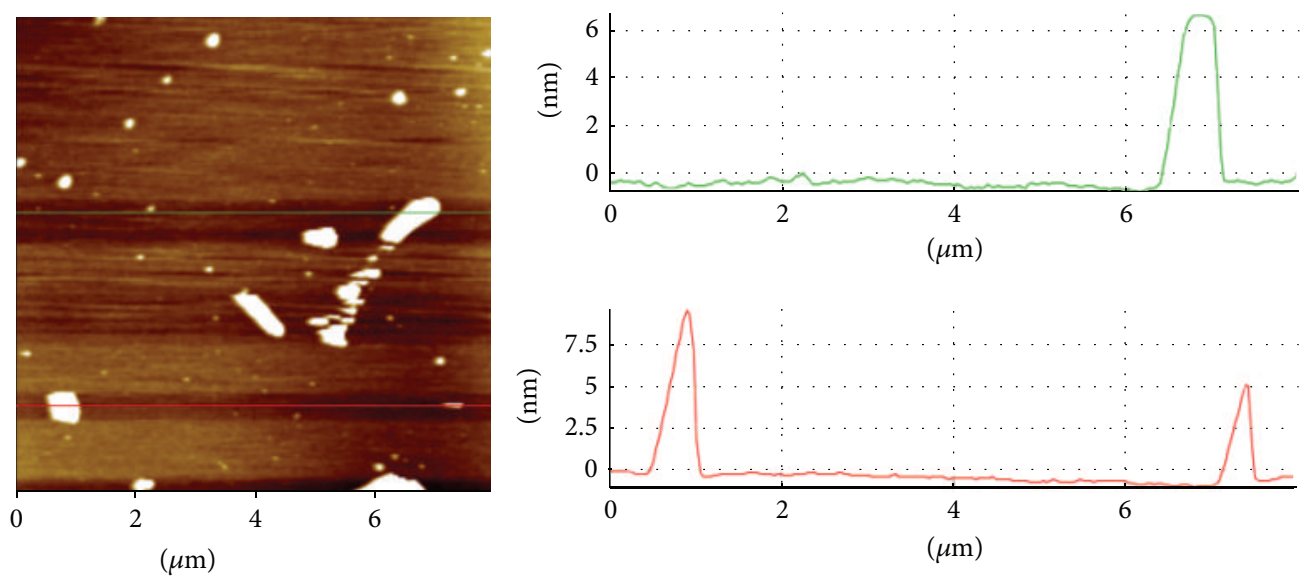

(a)
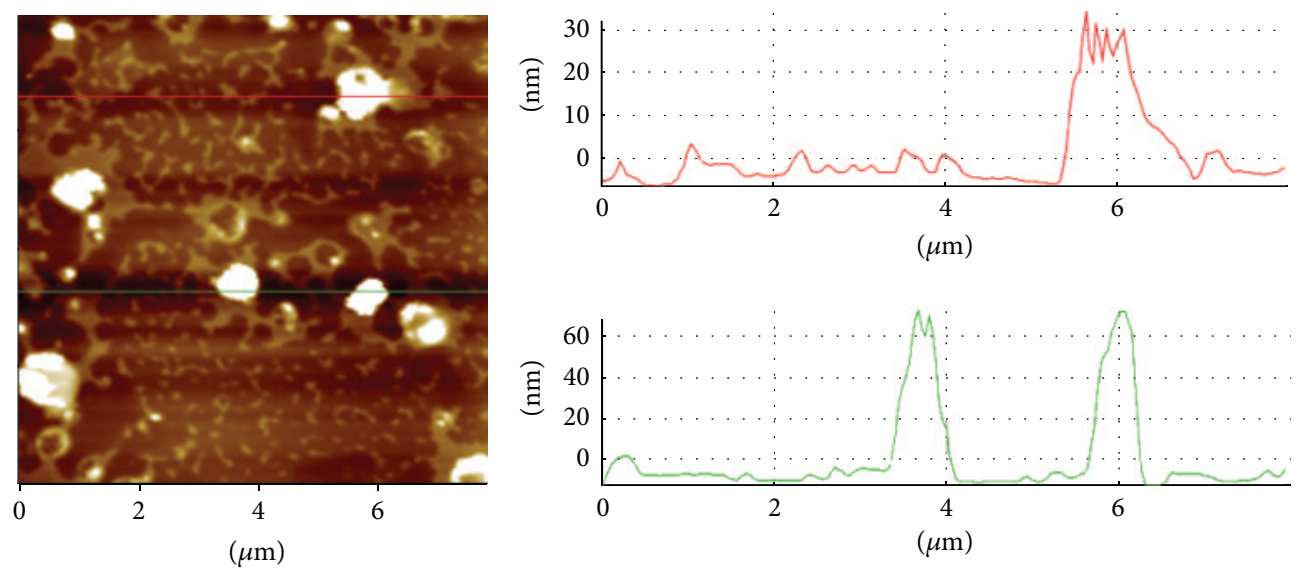

(b)

FIGURE 4: AFM images and height information of MPD micelles at $0.01 \mathrm{mM}$ (a) and $0.1 \mathrm{mM}(\mathrm{b})$. 


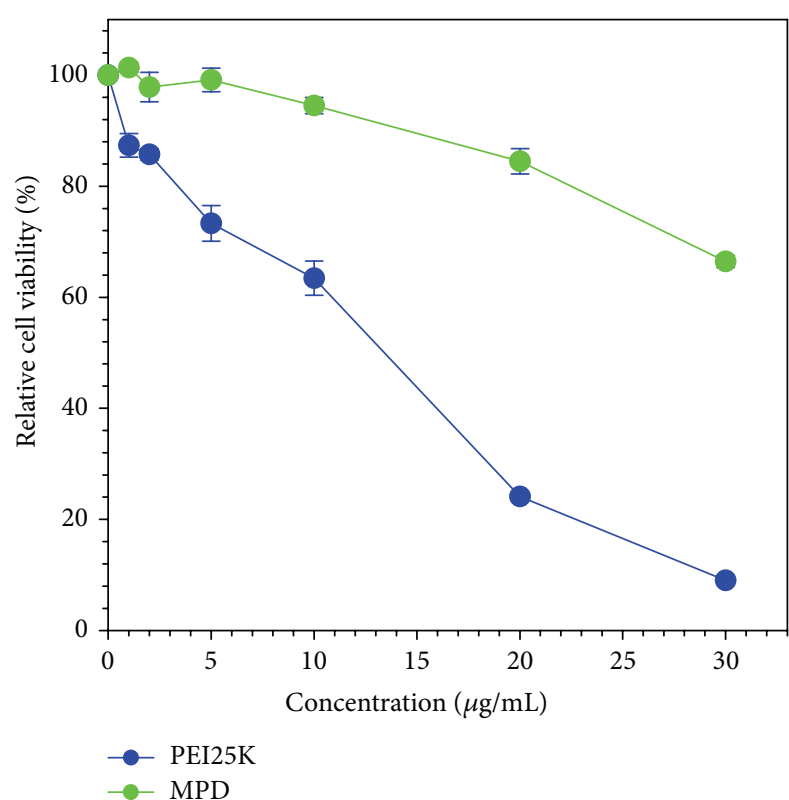

(a)

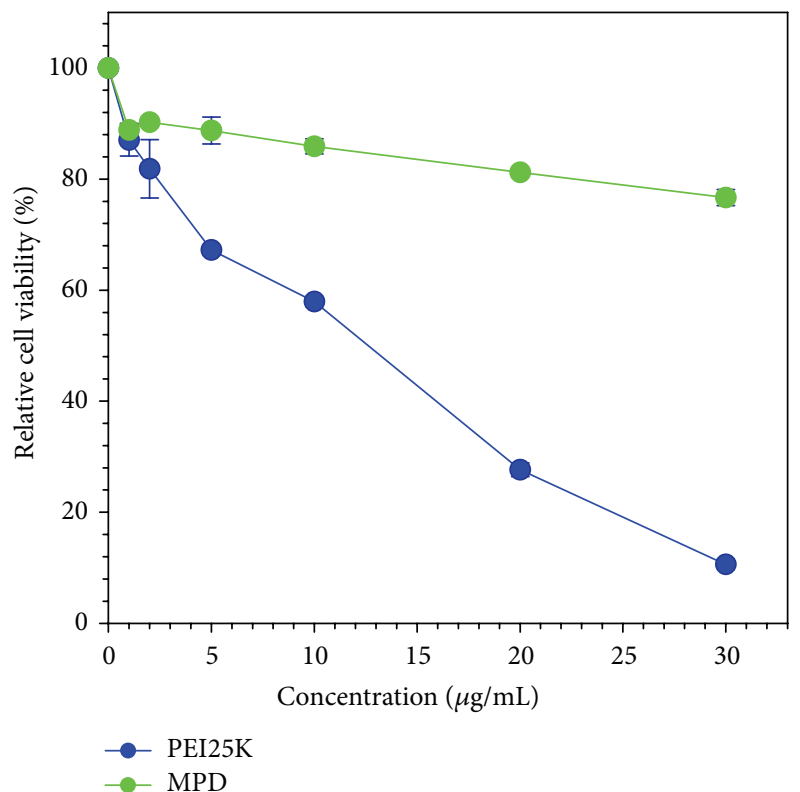

(b)

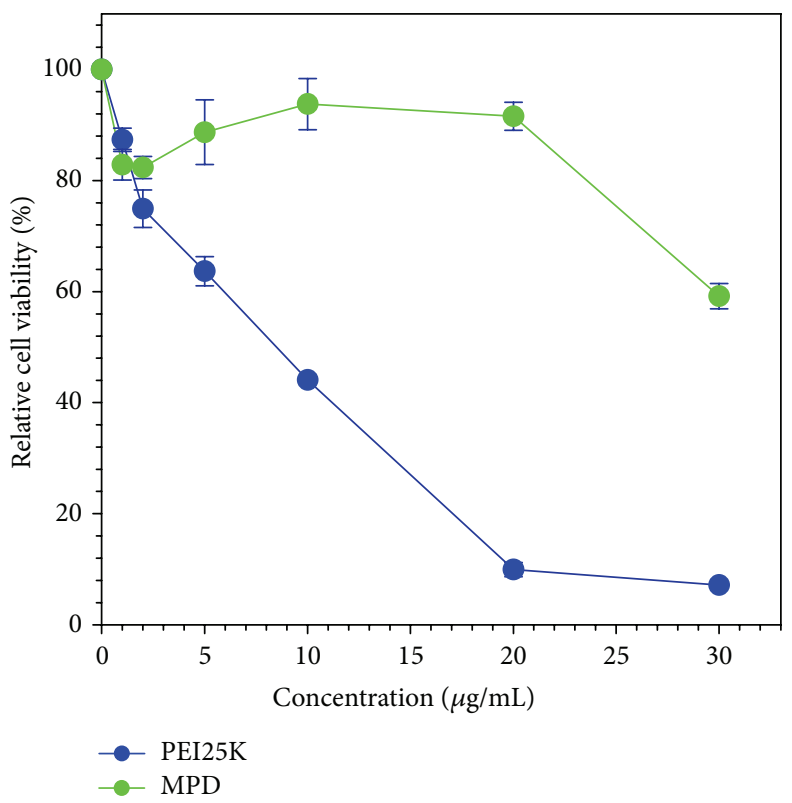

(c)

Figure 5: MTT assay results of MPD in A549 (a), C2C12 (b), and HEK293 (c) cells.

It is thought that MPD may possess the endosome buffering ability due to imidazole groups of histidine residues, of which $\mathrm{pKa}$ value is about 6. As shown in Figure 7, the transfection efficiency of PEI25k in Bafilomycin A1 condition was decreased to about 180 times lower value than normal condition. Similar to PEI25k, MPD also showed 20-170 times lower transfection efficiency in Bafilomycin A1 condition than normal condition. This result means that the transfection of MPD is mediated by endosomal escape by endosome buffering ability after cellular uptake.

\section{Conclusion}

Self-assembling multifunctional peptide was designed and dimerized for gene delivery systems. This MPD could self-assemble to micelle structures and condense pDNA by electrostatic interaction. The cytotoxicity of MPD was concentration-dependent but low in the examined concentration range. High transfection efficiency of MPD polyplexes in cancer cells expressing high level of MMP-2 showed the potential of MPD for targeted gene 


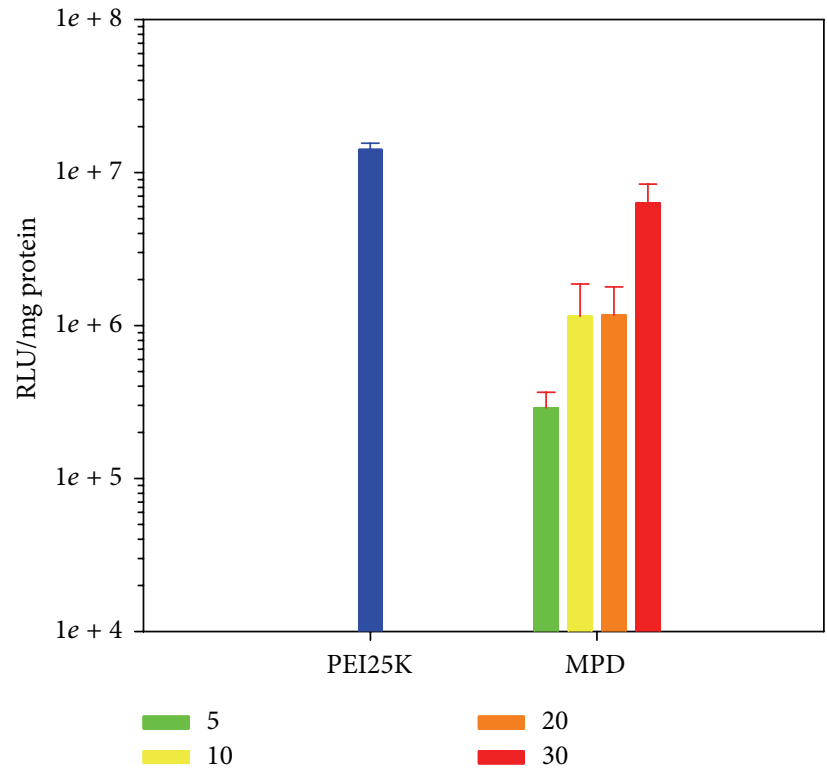

(a)

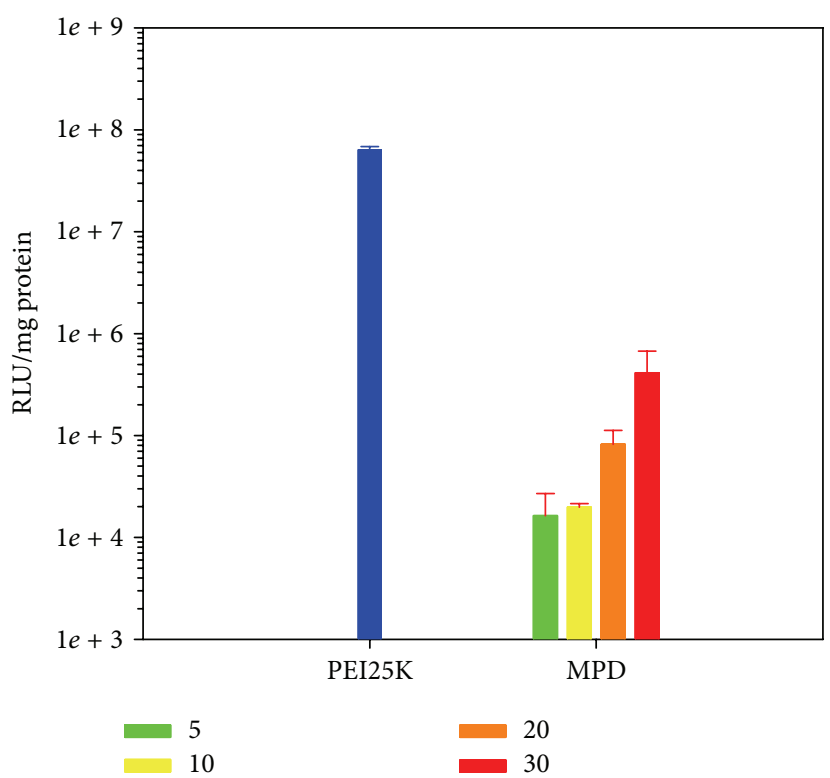

(c)

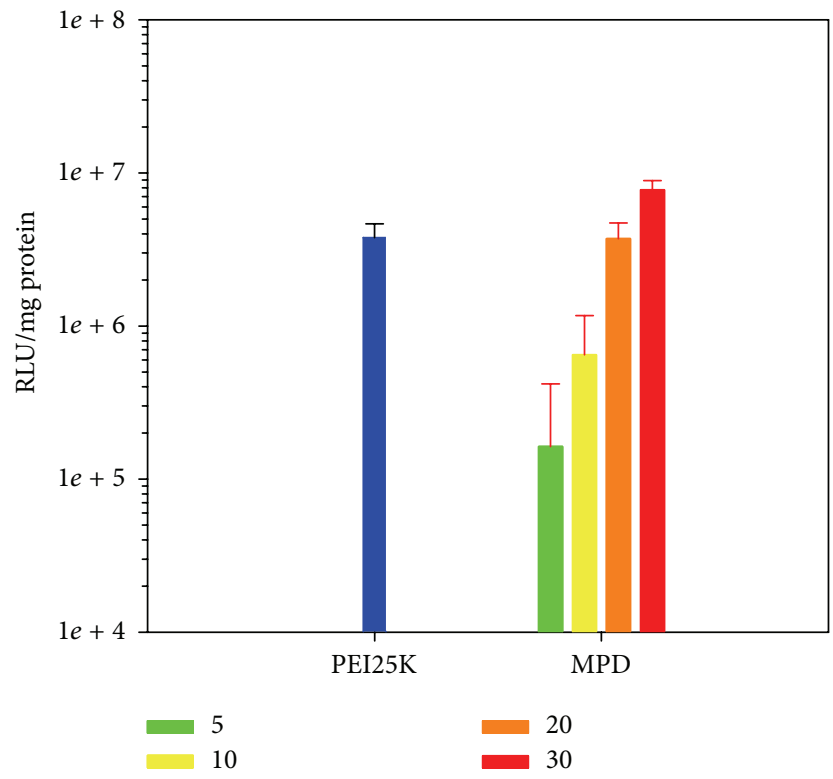

(b)

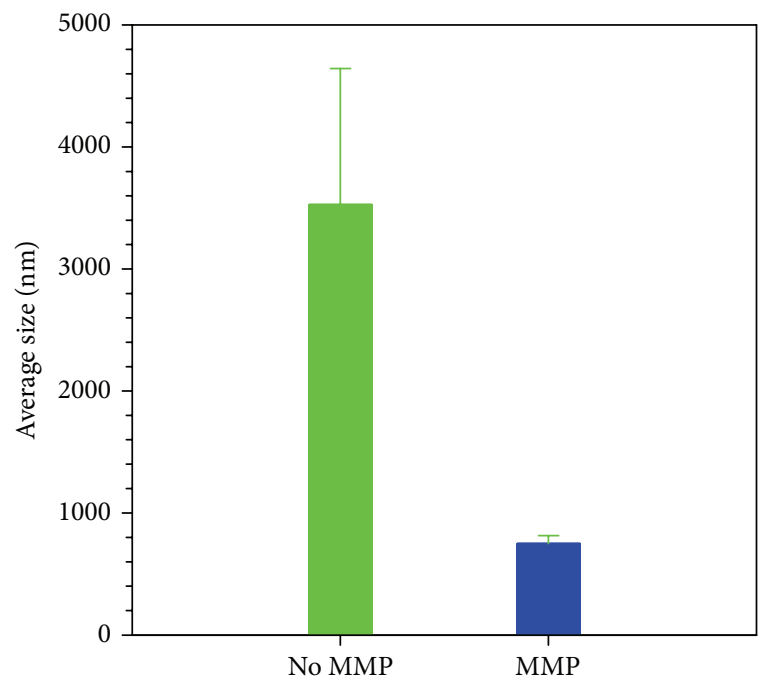

(d)

Figure 6: Transfection experiment results of MPD polyplexes in A549 (a), C2C12 (b), and HEK293 (c) cells. Numbers in boxes mean weight ratios of MPD polyplexes. PEI25k polyplexes were prepared at a weight ratio of 1. (d) Average sizes measurement result of MPD polyplexes (weight ratio: 20) in the absence or presence of MMP-2.

delivery. In addition, it was revealed that the transfection of MPD is mediated via endosomal escape by endosome buffering ability. In the further study, encapsulation of hydrophobic drug molecules in MPD micelles would be tried for synergistic effect of drug/gene codelivery for cancer therapy and the detailed mechanism for structural change of MPD polyplex by MMP cleavage also would be revealed.

\section{Conflict of Interests}

The authors declare that there is no conflict of interests regarding the publication of this paper.

\section{Acknowledgments}

This work was supported by the Basic Science Research Program through the National Research Foundation of Korea 


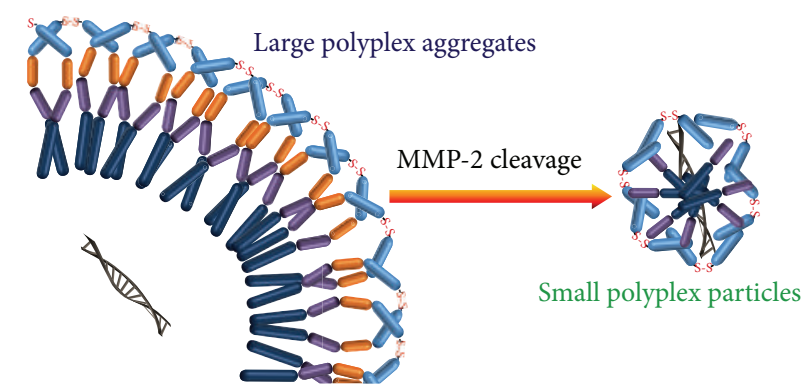

Cellular penetrating peptide moiety

MMP-2 specific sequence, structural change by MMP-2 cleavage

pH-responsive moiety, endosome buffering effect in endosomal $\mathrm{pH}$

$\Longrightarrow$ Hydrophobic moiety

Scheme 1: A scheme for the proposed mechanism of structural change of MPD polyplex in the presence of MMP-2.

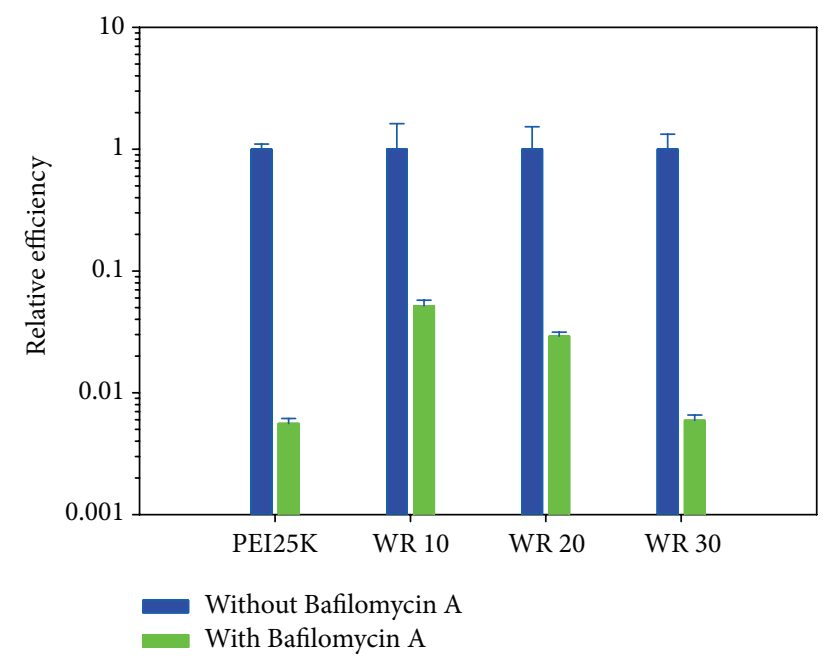

FIGURE 7: Transfection experiment results with/without Bafilomycin A1 in A549 cells. WR means the weight ratios of MPD polyplexes. Relative efficiency is the ratio of transfection efficiency with Bafilomycin Al to the transfection efficiency without Bafilomycin A1.

(NRF) funded by the Ministry of Education, Science and Technology (2011-0015045) and by the Ministry of Science, ICT, and Future Planning (NRF-2014R1A1A1037692). The authors also acknowledge Professor Yan Lee for permission to take Zetasizer measurements.

\section{References}

[1] H. O. Mccarthy, J. McCaffrey, C. M. Mccrudden et al., "Development and characterization of self-assembling nanoparticles using a bio-inspired amphipathic peptide for gene delivery," Journal of Controlled Release, vol. 189, pp. 141-149, 2014.

[2] S. Sundar, Y. Chen, and Y. W. Tong, "Delivery of therapeutics and molecules using self-assembled peptides," Current Medicinal Chemistry, vol. 21, no. 22, pp. 2469-2479, 2014.
[3] D. M. Copolovici, K. Langel, E. Eriste, and Ü. Langel, "Cellpenetrating peptides: design, synthesis, and applications," ACS Nano, vol. 8, no. 3, pp. 1972-1994, 2014.

[4] E. Ruoslahti, "RGD and other recognition sequences for integrins," Annual Review of Cell and Developmental Biology, vol. 12, pp. 697-715, 1996.

[5] D. Derossi, A. H. Joliot, G. Chassaing, and A. Prochiantz, "The third helix of the Antennapedia homeodomain translocates through biological membranes," The Journal of Biological Chemistry, vol. 269, no. 14, pp. 10444-10450, 1994.

[6] H. Brooks, B. Lebleu, and E. Vivès, "Tat peptide-mediated cellular delivery: back to basics," Advanced Drug Delivery Reviews, vol. 57, no. 4, pp. 559-577, 2005.

[7] M. Zhao and R. Weissleder, "Intracellular cargo delivery using Tat peptide and derivatives," Medicinal Research Reviews, vol. 24, no. 1, pp. 1-12, 2004.

[8] M. Zorko and Ü. Langel, "Cell-penetrating peptides: mechanism and kinetics of cargo delivery," Advanced Drug Delivery Reviews, vol. 57, no. 4, pp. 529-545, 2005.

[9] Y.-W. Won, P. P. Adhikary, K. S. Lim, H. J. Kim, J. K. Kim, and Y.-H. Kim, "Oligopeptide complex for targeted non-viral gene delivery to adipocytes," Nature Materials, vol. 13, pp. 1157-1164, 2014.

[10] A. Kiselev, A. Egorova, A. Laukkanen, V. Baranov, and A. Urtti, "Characterization of reducible peptide oligomers as carriers for gene delivery," International Journal of Pharmaceutics, vol. 441, no. 1-2, pp. 736-747, 2013.

[11] Y.-W. Won, H. A. Kim, M. Lee, and Y.-H. Kim, "Reducible poly(oligo-d-arginine) for enhanced gene expression in mouse lung by intratracheal injection," Molecular Therapy, vol. 18, no. 4, pp. 734-742, 2010.

[12] D. Soundara Manickam, H. S. Bisht, L. Wan, G. Mao, and D. Oupicky, "Influence of TAT-peptide polymerization on properties and transfection activity of TAT/DNA polyplexes," Journal of Controlled Release, vol. 102, no. 1, pp. 293-306, 2005.

[13] S. L. Lo and S. Wang, "An endosomolytic Tat peptide produced by incorporation of histidine and cysteine residues as a nonviral vector for DNA transfection," Biomaterials, vol. 29, no. 15, pp. 2408-2414, 2008.

[14] T.-I. Kim and S. W. Kim, "Bioreducible polymers for gene delivery," Reactive and Functional Polymers, vol. 71, no. 3, pp. 344-349, 2011.

[15] H. Margus, K. Padari, and M. Pooga, "Cell-penetrating peptides as versatile vehicles for oligonucleotide delivery," Molecular Therapy, vol. 20, no. 3, pp. 525-533, 2012.

[16] M. E. Stearns and M. Wang, "Type IV collagenase (Mr 72,000) expression in human prostate: benign and malignant tissue," Cancer Research, vol. 53, no. 4, pp. 878-883, 1993.

[17] G. Y. Lee, K. Park, S. Y. Kim, and Y. Byun, "MMPs-specific PEGylated peptide-DOX conjugate micelles that can contain free doxorubicin," European Journal of Pharmaceutics and Biopharmaceutics, vol. 67, no. 3, pp. 646-654, 2007.

[18] G. Gu, H. Xia, Q. Hu et al., "PEG-co-PCL nanoparticles modified with MMP-2/9 activatable low molecular weight protamine for enhanced targeted glioblastoma therapy," Biomaterials, vol. 34, no. 1, pp. 196-208, 2013.

[19] M. L. Read, S. Singh, Z. Ahmed et al., "A versatile reducible polycation-based system for efficient delivery of a broad range of nucleic acids," Nucleic Acids Research, vol. 33, no. 9, article e86, 2005. 
[20] T.-I. Kim, T. Rothmund, T. Kissel, and S. W. Kim, "Bioreducible polymers with cell penetrating and endosome buffering functionality for gene delivery systems," Journal of Controlled Release, vol. 152, no. 1, pp. 110-119, 2011.

[21] S. Son, K. Singha, and W. J. Kim, "Bioreducible BPEI-SS-PEGcNGR polymer as a tumor targeted nonviral gene carrier," Biomaterials, vol. 31, no. 24, pp. 6344-6354, 2010.

[22] G. Basu Ray, I. Chakraborty, and S. P. Moulik, "Pyrene absorption can be a convenient method for probing critical micellar concentration ( $\mathrm{cmc}$ ) and indexing micellar polarity," Journal of Colloid and Interface Science, vol. 294, no. 1, pp. 248-254, 2006.

[23] G. Lluri and D. M. Jaworski, "Regulation of TIMP-2, MT1-MMP, and MMP-2 expression during C2C12 differentiation," Muscle \& Nerve, vol. 32, no. 4, pp. 492-499, 2005.

[24] C. Chetty, S. S. Lakka, P. Bhoopathi, and J. S. Rao, "MMP-2 alters VEGF expression via $\alpha \mathrm{V} \beta 3$ integrin-mediated PI3K/AKT signaling in A549 lung cancer cells," International Journal of Cancer, vol. 127, no. 5, pp. 1081-1095, 2010.

[25] M. T. Malik and S. S. Kakar, "Regulation of angiogenesis and invasion by human pituitary tumor transforming gene (PTTG) through increased expression and secretion of matrix metalloproteinase-2 (MMP-2)," Molecular Cancer, vol. 5, article 61, 2006.

[26] A. Kichler, C. Leborgne, E. Coeytaux, and O. Danos, "Polyethylenimine-mediated gene delivery: a mechanistic study," Journal of Gene Medicine, vol. 3, no. 2, pp. 135-144, 2001.

[27] K. Kim, K. Ryu, and T.-I. Kim, "Cationic methylcellulose derivative with serum-compatibility and endosome buffering ability for gene delivery systems," Carbohydrate Polymers, vol. 110, pp. 268-277, 2014. 

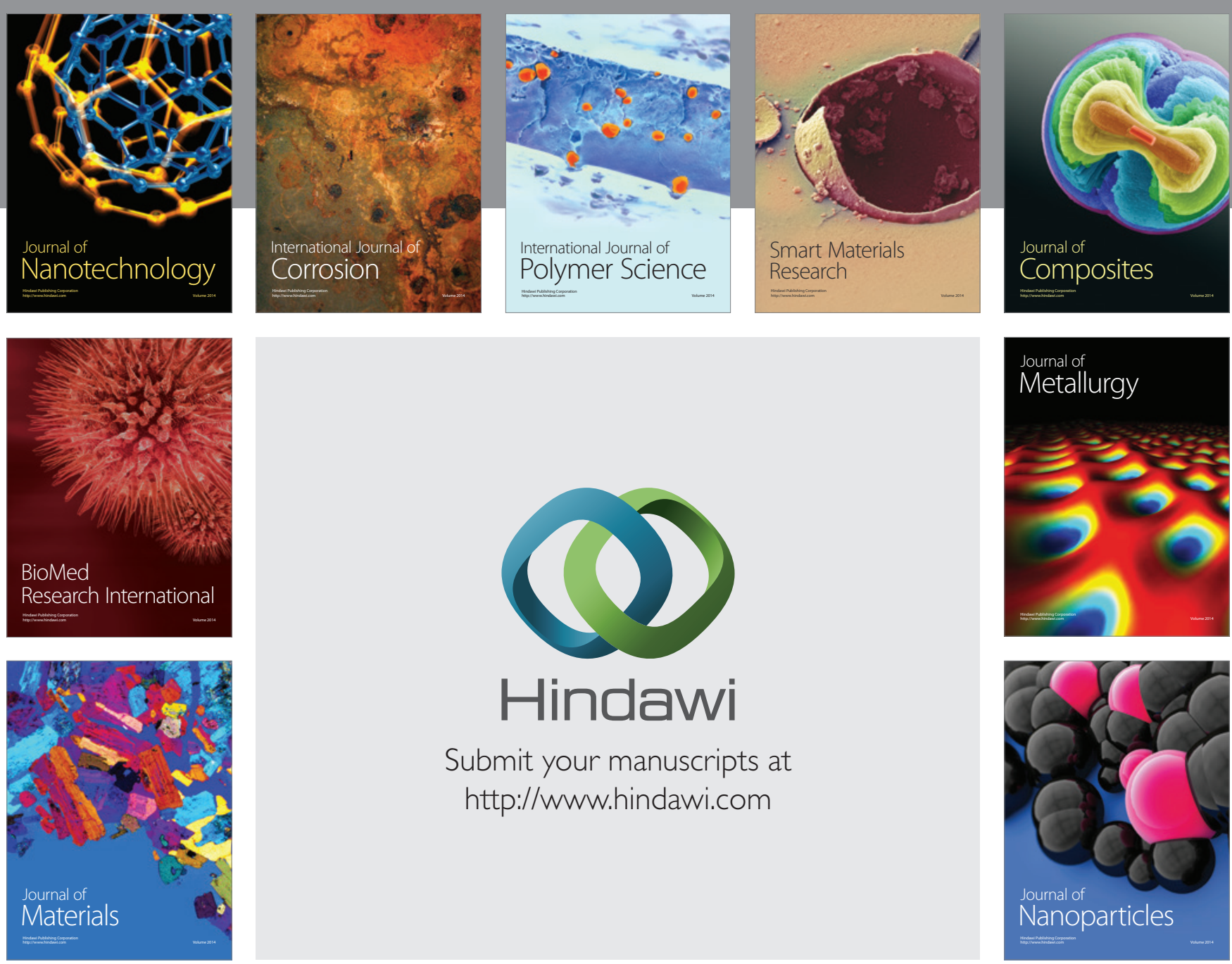

Submit your manuscripts at http://www.hindawi.com
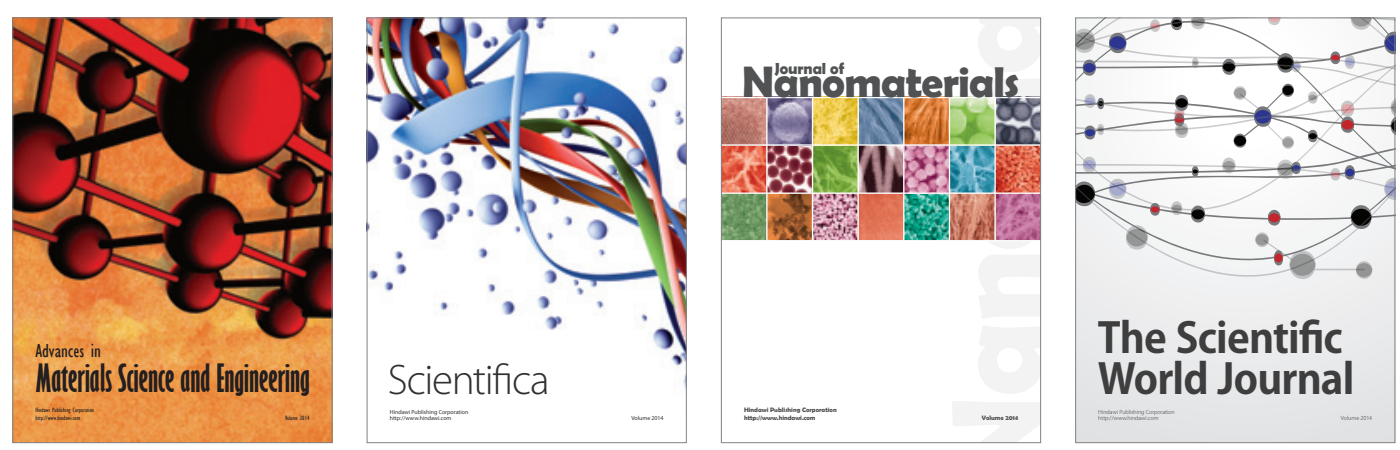

\section{The Scientific World Journal}
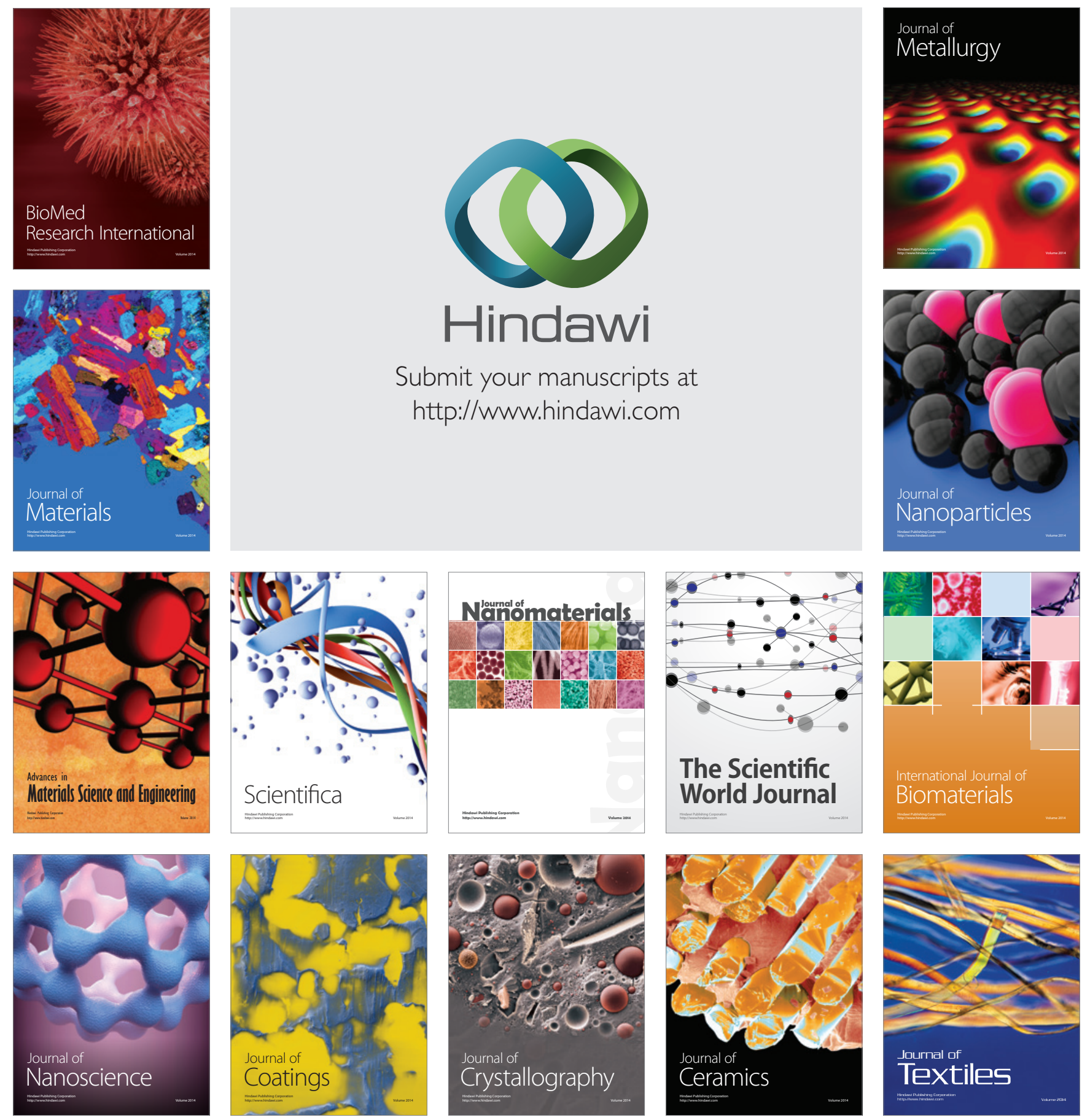